\title{
Higher-order ratios of baryon number cumulants
}

\author{
Bernd-Jochen Schaefer ${ }^{1,2 *}$ and Mathias Wagner $^{3 \dagger}$ \\ 1 Institut für Theoretische Physik, Justus-Liebig-Universität Gießen, Heinrich-Buff-Ring 16, 35392 Gießen, Germany \\ 2 Institut für Physik, Karl-Franzens-Universität Graz, Universitätsplatz 5, 8010 Graz, Austria \\ 3 Fakultät für Physik, Universität Bielefeld, Universitätsstraße, 33615 Bielefeld, Germany
}

\begin{abstract}
The relevance of higher order cumulants of net baryon number fluctuations for the analysis of freeze-out and critical conditions in heavy-ion collisions at LHC and RHIC is addressed. The sign structure of the higher order cumulants in the vicinity of the chiral crossover temperature might be a sensitive probe and may allow to elucidate their relation to the QCD phase transition. We calculate ratios of generalized quark-number susceptibilities to high orders in three flavor QCD-like models and investigate their sign structure close to the chiral crossover line.
\end{abstract}

PACS (2008): 12.38.Aw, 11.10.Wx , 11.30.Rd, 12.38.Gc

Keywords: General properties of QCD • Chiral symmetries • Finite-temperature field theory

(C) Versita Warsaw and Springer-Verlag Berlin Heidelberg.

\section{Introduction}

One of the distinctive feature of the QCD phase diagram is the possible emergence of a critical endpoint (CEP) where the first-order chiral phase transition line at large chemical potential terminates. Many properties of the endpoint such as, e.g., its precise location are still unknown. However, for experiments that search for this point the knowledge of its characteristics is inevitable. Since the expected transition is of second-order at this point possible signatures are based on the singular behavior of the thermodynamic functions in its vicinity. Recently, it was pointed out that event-by-event fluctuations of particle multiplicities and their nonmonotonic behavior might serve as a probe to locate a possible CEP in the phase diagram. By scanning the center of mass energy and thus the baryochemical potential an increase and then a decrease in the number fluctuations of, e.g., pions and protons should be seen as one crosses the critical point. Unfortunately, in a realistic heavy-ion collision the expected signals are washed out due to the critical slowing down phenomenon and finite volume effects. Furthermore, correlations can only build up for a finite time in the colliding system and consequently the correlations length

* E-mail: bernd-jochen.schaefer@uni-graz.at

$\dagger$ E-mail: mwagner@physik.uni-bielefeld.de 
is cut off. Some conservative estimates yield an increase of the correlation length only by a factor 3 as the system crosses the critical point. Therefore, more sensible quantities are needed for the analysis of the freeze-out and critical conditions in heavy-ion collisions. For this reason higher-order cumulants or ratios of higher-order generalized susceptibilities have been suggested as suitable quantities because they depend on higher powers of the correlation length [1].

\section{A three-flavor model analysis}

Fluctuations of conserved charges are quantified by cumulants in statistics and are related to generalized susceptibilities [2]. They are defined as derivatives of the logarithm of the partition function with respect to the appropriate chemical potentials. For three different chemical potentials we have accordingly

$$
\chi_{n_{i}, n_{j}, n_{k}} \equiv \frac{1}{V T} \frac{\partial^{n_{i}}}{\partial\left(\mu_{i} / T\right)^{n_{i}}} \frac{\partial^{n_{j}}}{\partial\left(\mu_{j} / T\right)^{n_{j}}} \frac{\partial^{n_{k}}}{\partial\left(\mu_{k} / T\right)^{n_{k}}} \log Z
$$

where $n_{i}, \ldots$ denotes the order of the derivatives and the indices $(i, j, k)=(u, d, s)$ the quark flavor. The generalized susceptibilities evaluated at vanishing $\mu_{f}$ are the Taylor expansion coefficients of the pressure series in powers of $\mu_{f} / T$. The partition function is evaluated in a renormalized three-flavor Polyakov-quark-meson (PQM) model with an axial $U(1)_{A}$ symmetry breaking term and a logarithmic Polyakov-loop potential in meanfield approximation where the ultraviolet divergent fermion vacuum contribution has been taken into account [3]. Thus, fermion fluctuations are taken into account whereas meson and Polyakov-loop fluctuations are still ignored. The resulting phase diagram in comparison with the one obtained in the three-flavor quark-meson model is shown in Fig. 1

\subsection{Ratios of baryon number moments}

In the following we focus on one uniform quark chemical potential and denote the $n$th to $m$ th order moment ratio of the quark number fluctuations $\chi_{n}$ as

$$
R_{n, m} \equiv \chi_{n}(T, \mu) / \chi_{m}(T, \mu)
$$

The evaluation of the ratios has been automated by using algorithmic differentiation techniques [4]. The kurtosis $R_{4,2}$ basically measures the quark content of particles carrying baryon number and has been calculated in the vicinity of the crossover region of the phase diagram. In the hadron resonance gas (HRG) model the ratio is temperature independent and all moments stay positive since the HRG model has no singularities. Thus, any deviation from the HRG model result might be an indicator for a real critical phenomenon even if the lower-order moments and the thermodynamics including particle yields are well described by the HRG model [5]. In our model calculation the kurtosis becomes negative and consequently a negative region around the crossover line emerge in the phase diagram. Note that these three-flavor results differ from a corresponding two-flavor PQM mean-field 


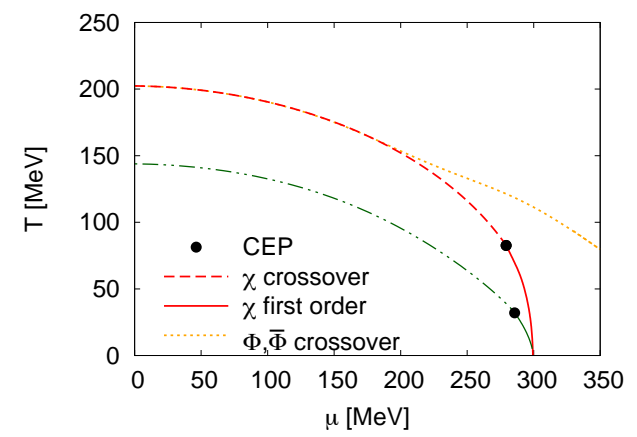

Figure 1. Phase diagrams for three flavor of the renormalized PQM model with a running $T_{0}$ parameter in the logarithmic Polyakov-loop potential and of the quark-meson model. Dashed lines denote the chiral crossover, solid lines the firstorder chiral transition and dotted lines the peak in the temperature-derivative of the Polyakov-loops.

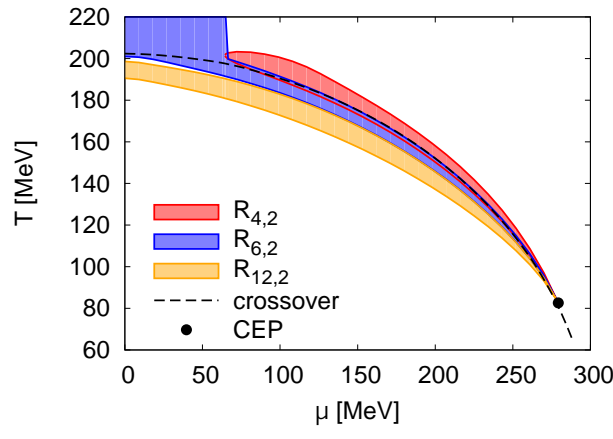

(a) Polyakov-quark-meson model

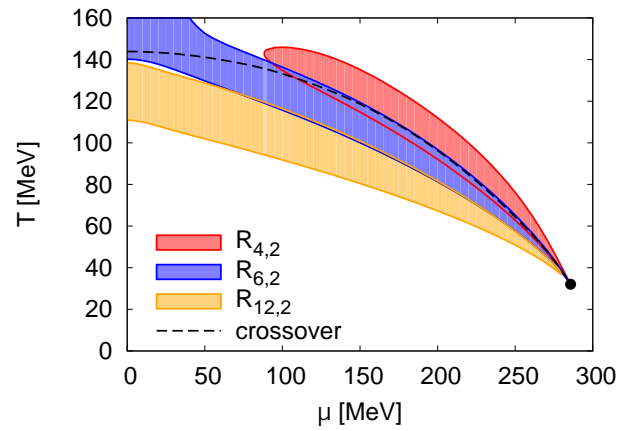

(b) Quark-meson model

Figure 2. Three negative areas of $R_{n, 2}$ ratios close to the chiral crossover line in both models.

calculation with and without the fermion vacuum term in the grand potential. Without the vacuum term in the two-flavor case the kurtosis $R_{4,2}$ develops a peak at zero chemical potential near the crossover temperature which is a remnant of the first-order transition in the chiral limit, see [6] for more details. The inclusion of the fermion vacuum term changes the transition to second-order in the chiral limit which is consistent with universality arguments [7]. However, for three massless flavor a first-order transition always emerges in the chiral limit with or without the fermion vacuum term. The peak of the kurtosis for physical pion masses is already less pronounced if the vacuum term is neglected and finally vanishes in the three-flavor renormalized model. Thus, the suppression of the kurtosis peak at $\mu=0$ must be caused by the strange quarks and not by fluctuations $[3,7]$.

\subsection{Sign structure of higher-order ratios close to the transition}

Higher-order ratios behave in a similar way. They oscillate within a narrow temperature interval for temperatures close to the chiral transition. Generically, the structures of all moments at $\mu=0$ are related to each other and the behavior including the amplitudes of $\chi_{n}$ can be deduced by the temperature derivative of the preceding $\chi_{n-2}$ [8]. In contrast to the HRG model all higher-order ratios become negative in the vicinity of the crossover line for 
nonvanishing chemical potential as shown in Fig. 2. In this figure the negative regions of three even ratios $R_{n, 2}$ in the renormalized three-flavor PQM model along the chiral crossover line are compared to a corresponding three-flavor renormalized QM model without the Polyakov-loop. All negative regions are closely correlated with the crossover curve and converge exactly at the CEP. For $n>4$ they are shifted slightly in the hadronic phase. The Polyakov loop sharpens these negative regions around the chiral transition. In the renormalized models the CEP is pushed towards higher chemical potentials by the inclusion of the vacuum terms but the curvature of the crossover line seems not to be changed. However, the crossover is washed out by fluctuations which yields larger negative regions. In summary the behavior of the negative regions can surely be attributed to critical dynamics [9]. The findings underline once more the importance of fluctuations: all regions calculated in the renormalized models are shifted more in the hadronic phase.

The knowledge of how the negative regions evolve towards the endpoint might be used to construct new criteria to improve the critical temperature estimate from a Taylor expansion around $\mu=0$. For this reason it is instructive to define the distance $\Delta T=T_{n}-T_{\chi}$ of the first zero in temperature direction of the ratio $R_{n, 2}$ to the crossover temperature $T_{\chi}$. In the left panel of Fig. 3 the distance $\Delta T$ of several even ratios $R_{n, 2}$ is shown as a function of $\mu / T$. Only the ratio $R_{4,2}$ remains positive away from the CEP. For all higher-order ratios the first zero in $R_{n, 2}$ is pushed into the hadronic phase and all $\Delta T$ are negative already for $\mu / T \sim 0$. They obey a minimum whose precise location and depth is model dependent. With the Polyakov loop the transition is sharper and the minima are not as deep. Remarkable is the almost linear behavior of $\Delta T$ for intermediate $\mu / T$ ratios in the PQM and QM models for all ratios $R_{n, 2}$. The linear extrapolation of $\Delta T$ from intermediate $\mu / T$ to larger values where $\Delta T$ vanishes might serve as an estimator for the proximity of the thermal freeze-out to the crossover line and the existence of a possible endpoint can be ruled out for smaller values of $\mu / T$. This estimate could be strengthened by considering only the difference of the subsequent roots in the odd ratios which is independent of the knowledge of the chiral crossover temperature. The relative temperature distance $\Delta \tau=T_{n+2}-T_{n}$ for several even ratios $R_{n, 2}$ is shown as a function of $\mu / T$ in the right panel of Fig. 3. The curves exhibit a similar behavior as in the previous figure except the ordering of the curves with respect to $n$ is opposite. With increasing $n$ the distance between subsequent ratios decreases, signaling a possible convergence of the negative regions in the phase diagram. An estimation of the lower bound of the CEP with a linear fit is in between $15 \%$ of the actual CEP model values.

\section{Conclusion}

Higher-order cumulants or generalized susceptibilities are more sensitive on the diverging correlation length and are promising quantities for the experimental search of an endpoint in the QCD phase diagram. In our model analysis the higher-order moments differ significantly from the HRG model results along the freeze-out curve due to the existence of an endpoint. A region with negative values of the ratios of momenta emerges close to the chiral transition line. They are shifted towards the hadronic phase and converge at the endpoint. In order to 

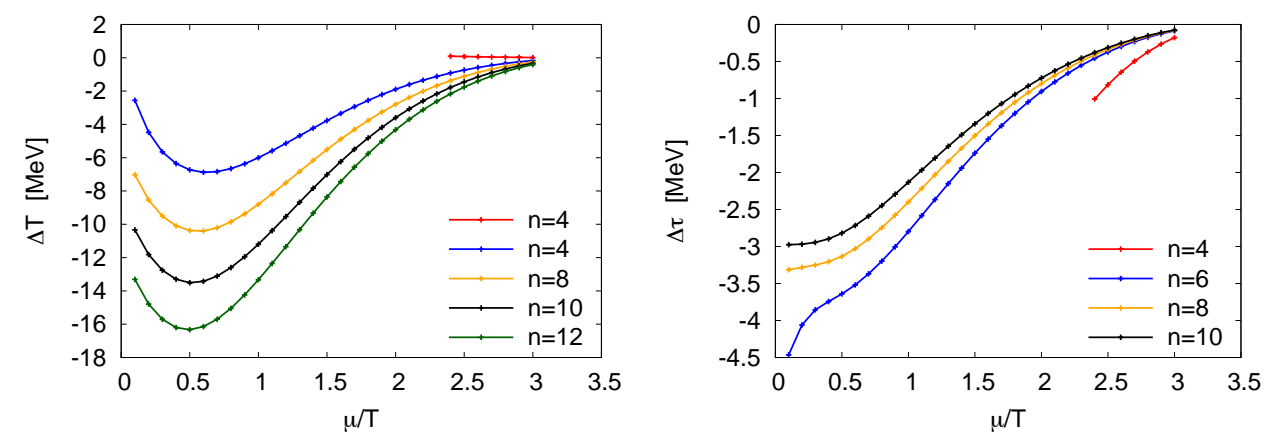

Figure 3. Distance $\Delta T=T_{n}-T_{\chi}$ (left) and the relative distance $\Delta \tau=T_{n+2}-T_{n}$ (right) of the first zero for various ratios $R_{n, 2}$ for even integers $n$ as a function of $\mu / T$ in the renormalized PQM model. $T_{\chi}$ denotes the chiral critical temperature.

quantify this general trend we have introduced the distance of the first zero in temperature direction of various ratios to the crossover temperature and the relative distance between subsequent roots which is independent of the insecure chiral crossover temperature. By using linear extrapolations as estimators we could rule out the existence of an endpoint for smaller $\mu / T$ ratios.

\section{References}

[1] M. A. Stephanov, K. Rajagopal, and E. V. Shuryak, Phys. Rev. D60, 114028 (1999); L. Kumar, Nucl.Phys. A862-863, 125 (2011); B. Berdnikov and K. Rajagopal, Phys. Rev. D61, 105017 (2000); X. Luo, B. Mohanty, H. G. Ritter, and N. Xu, arXiv:1105.5049 [nucl-ex] ; M. A. Stephanov, Phys. Rev. Lett. 102, 032301 (2009);

C. Athanasiou, K. Rajagopal, and M. Stephanov, Phys. Rev. D82, 074008 (2010).

[2] V. Koch, arXiv:0810.2520 [nucl-th].

[3] B.-J. Schaefer and M. Wagner, Phys.Rev. D85, 034027 (2012).

[4] M. Wagner, A. Walther, and B.-J. Schaefer, Comp. Phys. Commun. 181, 756 (2010).

[5] A. Andronic, P. Braun-Munzinger, and J. Stachel, Acta Phys.Polon. B40, 1005 (2009); B. Friman, F. Karsch, K. Redlich, and V. Skokov, Eur. Phys. J. C71, 1694 (2011).

[6] E. Nakano, B.-J. Schaefer, B. Stokic, B. Friman, and K. Redlich, Phys.Lett. B682, 401 (2010).

[7] V. Skokov, B. Friman, E. Nakano, K. Redlich, and B.-J. Schaefer, Phys. Rev. D82, 034029 (2010).

[8] B.-J. Schaefer, M. Wagner, and J. Wambach, PoS CPOD2009, 017 (2009); Phys. Rev. D81, 074013 (2010); J. Wambach, B.-J. Schaefer, and M. Wagner, Acta Phys. Pol. B Proc. Suppl. 3, 691 (2010); F. Karsch, B.-J. Schaefer, M. Wagner, and J. Wambach, Phys.Lett. B698, 256 (2011).

[9] V. Skokov, B. Friman, and K. Redlich, Phys.Lett. B708, 179 (2012); F. Karsch and K. Redlich, Phys.Lett. B695, 136 (2011). 